\title{
Die Dogmatik des menschenrechtlichen Gleichheitsschutzes
}

\author{
Stefan Huster"
}

Rezension zu Tilmann Altwicker, Menschenrechtlicher Gleichheitsschutz, Heidelberg u.a., Springer-Verlag (Beiträge zum ausländischen öffentlichen Recht und Völkerrecht, Bd. 223) 2011, XXX und 549 S., 99,95 €; ISBN: 978-3-642-18199-3

\section{Der wachsende Bedarf an EMRK-Dogmatik}

Ein kundiger Kollege hat kürzlich anlässlich der Entscheidungen zur Sicherungsverwahrung die „selbstbewusste und expansive Rechtsprechung“ des EGMR beklagt und deren Sinnhaftigkeit angesichts des ausgebauten Grundrechtsschutzes in Deutschland und vielen anderen Mitgliedstaaten des Europarats in Frage gestellt: Sie sorge dort „nicht mehr für den menschenrechtlich gebotenen Mindeststandard oder überhaupt nur für ein besseres Konzept des Grundrechtsschutzes, sondern ersetzt in vielen Fällen einfach das bestehende System durch irgendein anderes“ (Volkmann, JZ 2011, S. 835, 841f.). Unstreitig dürfte jedenfalls sein, dass die EMRK in den letzten Jahren erheblich an Bedeutung gewonnen hat und der geplante Beitritt der Europäischen Union zur EMRK diese Entwicklung verstärken wird - wie man dann auch immer das Verhältnis von EMRK, Unionsrecht und Grundgesetz und von EGMR, EuGH und BVerfG im „europäischen Verfassungsgerichtsverbund“ konstruieren mag. Es besteht daher ein zunehmender Bedarf an dogmatischer Aufarbeitung der EMRK und der Rechtsprechung des EGMR. Neben EMRK-Kommentaren (nach Frowein/Peukert und Meyer-Ladewig jetzt auch Karpenstein/Mayer), Lehrbüchern zur EMRK (vgl. Grabenwarter bzw. jetzt Grabenwarter/Pabel und Anne Peters) und Konkordanzkommentaren (vgl. Grote/Marauhn) sind daher auch monographische Aufarbeitungen der Dogmatik von großem Interesse.

Die umfangreiche Arbeit von Altwicker, entstanden aus einer an der Universität Mannheim eingereichten und von Hans-Joachim Cremer betreuten Dissertation, nimmt sich insoweit der Gleichheitsrechte der EMRK an. Dies lassen Titel und Anlage der Arbeit nicht gleich erkennen, aber in der Sache geht es um eine Systematisierung der Gleichheitsrechtsprechung des EGMR, die im zentralen und weitaus umfangreichsten dritten Teil der Arbeit (S. 121 - 396) dargestellt und analysiert wird. Eingekleidet wird diese Rekonstruktion durch einleitende Bemerkungen (S. 1 - 6), begriffliche Vorbemerkungen zum Gleichheitsbegriff (1. Teil, S. 7 - 25), einen Überblick zum menschenrechtlichen Gleichheitsschutz (2. Teil, S. 27 - 119) und eine „rechtsethische“ Aufarbeitung der Prinzipien, die die Gleichheitsrechtsprechung anleiten (4. Teil, S. 397 - 488). Eine Betrachtung zum „Problem des Endes“ (S. 489 494) beschließt das Werk.

* Prof. Dr. Stefan Huster lehrt Staats- und Verwaltungsrecht und Sozialrecht an der Ruhr-Universität Bochum. 


\section{Theorie und Praxis der menschenrechtlichen Gleichheit}

1. Die Arbeit setzt ein mit begrifflichen Vorklärungen zum Gleichheitsbegriff. Dies ist zu begrüßen, weil viele juristische Diskussionen über Fragen der Gleichbehandlung daran kranken, dass schon auf einer sehr grundsätzlichen Ebene, die mit dem Rechtsbegriff der Gleichheit noch gar nichts zu tun hat, begriffliche Konfusion herrscht. Dass man präskriptive und deskriptive Gleichheitsurteile („X und Y sind gleich“ vs. „X und Y sollten gleichbehandelt werden bzw. gleich sein“), die Gleichheit von Behandlungen und von Zuständen („X und Y werden gleich behandelt" vs. „X und $\mathrm{Y}$ werden so behandelt, dass sie über die gleichen Ressourcen o.ä. verfügen“ - was ja bekanntlich typischerweise nur durch eine ungleiche Behandlung erreicht werden kann) sowie Gleichbehandlung im deskriptiven und normativen Sinne („X und $\mathrm{X}$ werden identisch behandelt" vs. „X und $\mathrm{Y}$ werden gerecht behandelt") auseinanderhalten sollte, sieht Altwicker ebenso zutreffend wie den Umstand, dass die allgemeine Gleichheitsforderung, dass Gleiches gleich zu behandeln ist, der Aufladung durch Gerechtigkeitsmaßstäbe bedarf, wenn sie nicht leer bleiben soll. Exemplarisch erwähnt Altwicker drei prominente Ansätze der zeitgenössischen politischen Philosophie (Rawls' Grundgüter, Dworkins Ressourcen und Sens Fähigkeiten), die beanspruchen, eine plausible Theorie der relevanten Gleichheitshinsicht zu entwickeln.

2. Die Arbeit wendet sich sodann dem menschenrechtlichen Gleichheitsanspruch, wie er in völkerrechtlichen Normen verankert ist, auf einer sehr grundsätzlichen Ebene zu. Altwicker geht dabei von der Beobachtung aus, dass dieser Gleichheitsanspruch zwei Ausprägungen gefunden hat: als allgemeines Gleichheitsrecht (wie in Art. 26 IPpbR) und als Diskriminierungsverbot (wie in Art. 14 und Art. 1 ZP 12 EMRK). Als gemeinsamen „Fluchtpunkt“ dieser Ausprägungen sieht er ein objektives Prinzip der Rechtsgleichheit im Völkerrecht an, das er zunächst auf seine Bedeutung, seine Quellen und seine Funktionen hin näher untersucht.

Mit Blick auf das allgemeine Gleichheitsrecht als erste subjektivrechtliche Ausprägung des Prinzips der Rechtsgleichheit stellt Altwicker zutreffend heraus, dass dessen übliche zweistufige Dogmatik („Liegt eine Ungleichbehandlung vor? Ist diese gerechtfertigt? ") mancherlei Probleme mit sich bringt. Das von dem Rezensenten entwickelte Modell der Gleichheitsprüfung, das sich der Eingriffsdogmatik der Freiheitsrechte annähert, wird als dogmatischer Fortschritt gewürdigt, während der Ansatz von Somek, den allgemeinen Gleichheitssatz auf ein Diskriminierungsverbot zu reduzieren, zurückgewiesen wird.

Das eigentliche Interesse von Altwicker gilt allerdings dem Antidiskriminierungsmodell der Rechtsgleichheit. Dieses wird zunächst in sehr abstrakter Form eingeführt, bevor es im folgenden Teil anhand der Rechtsprechung des EGMR detailliert analysiert wird. 
3. Dieser dritte und zentrale Teil der Arbeit mit einem Umfang von fast 300 Seiten stellt bereits in sich eine dissertationsreife Leistung auf außerordentlichen hohem Niveau dar. Auch wenn man nicht mit allen Ergebnissen einverstanden ist, ist es bemerkenswert, mit welcher Akribie und Umsicht das Rechtsprechungsmaterial hier systematisiert wird, zumal ähnlich umfangreiche Untersuchungen dazu noch nicht vorliegen. Fast ist man versucht zu sagen, dass dieses Kapitel des Buches gegenüber seinen anderen Teilen derartig heraussticht, dass es Altwicker dabei hätte bewenden lassen sollen.

Altwicker schichtet einen „allgemeinen Teil“ der antidiskriminierungsrechtlichen Dogmatik, der die Anwendungsbedingungen und die allgemeinen dogmatischen Strukturen der Art. 14 und 1 ZP 12 EMRK betrifft, von einem „,besonderen Teil“ $\mathrm{ab}$, in dem die einzelnen Wirkungsrichtungen des Diskriminierungsverbots herausgearbeitet werden. Ob diese Unterteilung besonders glücklich ist, ist zweifelhaft; so dürften sich die Rechtfertigungs- und Beweislastanforderungen für Ungleichbehandlungen ( „allgemeiner Teil“) danach unterscheiden, ob eine direkte, indirekte, passive oder positive Diskriminierung („,besonderer Teil“) vorliegt. In der Sache sind die Ausführungen aber immer verständig und diskussionswürdig, wie im Folgenden nur anhand einiger Beispiele angedeutet werden kann. So kritisiert Altwicker etwa überzeugend die - auch in der Rechtsprechung des EGMR zu beobachtende - Unsitte, der eigentlichen Gleichheitsprüfung gelegentlich die Frage nach der „Vergleichbarkeit" vorzuschalten. Da man alles miteinander vergleichen kann, werden hier nur Wertungen eingeschmuggelt, die sehr viel transparenter in der Rechtfertigungsprüfung abgearbeitet werden sollten.

Ein gewisses methodisches Problem zeigt sich in den Ausführungen zu der wichtigen Frage, welche Differenzierungsgründe vom EGMR als „verdächtig“ angesehen werden (sollten). Altwicker will hier naheliegender Weise vom Schutzzweck der Diskriminierungsverbote aus argumentieren, sieht sich dafür aber gezwungen, auf die rechtsphilosophische Rekonstruktion im 4. Teil seiner Arbeit zu vorauszuverweisen. Dies ist ein Indiz dafür, dass die dogmatischen und rechtsethischen Aspekte enger miteinander zusammenhängen, als uns der Aufbau seiner Arbeit suggeriert. Er wendet sich dann den zentralen Fragen der Intensität der Rechtfertigungsprüfung, dem Beurteilungsspielraum und den Darlegungs- und Beweislasten zu. Wiederum ist der Leser beeindruckt, wie übersichtlich und mit hohem Abstraktionsniveau, gleichzeitig aber sensibel gegenüber dem Rechtsprechungsmaterial argumentiert wird. Und doch bleibt ein kleines Unbehagen, auf das noch zu sprechen zu kommen sein wird: Sollte es nicht für die dogmatische Analyse eine Rolle spielen, dass hier die Rechtsprechung eines Menschengerichtshofs und nicht eines nationalen Verfassungsgerichts untersucht wird? 
Der „besondere Teil“ der antidiskriminierungsrechtlichen Dogmatik will im Kern vier Verbote unterscheiden: der direkten, der indirekten, der passiven und der positiven Diskriminierung. Die direkte Diskriminierung, die unmittelbar an ein Diskriminierungsmerkmal anknüpft, ist ein bekanntes Rechtsproblem, während die EGMR-Rechtsprechung zur positiven Diskriminierung kaum etwas hergibt (zumal deren Rechtsproblem dann ja regelmäßig die direkte Diskriminierung der Benachteiligten ist), so dass sich die interessantesten Fragen mit Blick auf die indirekte und die passive Diskriminierung stellen. Dabei können auch komplizierte Abgrenzungsfragen auftreten: Ist die Nicht-Berücksichtigung religiöser Sonderbedürfnisse eine Form der direkten Diskriminierung in Form der Gleichbehandlung des Ungleichen oder handelt es sich um eine indirekte Diskriminierung aufgrund der gruppenspezifischen Auswirkung? Altwicker will die erste Variante vertreten und findet dafür auch Belege in der Rechtsprechung. Allerdings ist es zweifelhaft, ob sich das durchhalten lässt, wenn die Anknüpfung gleichzeitig konstitutives Merkmal der direkten Diskriminierung sein soll.

Positive Handlungspflichten des Staates, die in dieser Stringenz aus der nationalen Gleichheitsdogmatik nicht bekannt sind, sollen sich aus dem Verbot der passiven Diskriminierung ergeben. Im Anschluss an und in Auseinandersetzung mit der menschenrechtsdogmatischen Systematisierung „respect - protect - fulfill“ sieht Altwicker insoweit drei Gewährleistungsdimensionen: eine Schutzpflicht gegenüber privater Diskriminierung, eine Gewährleistungspflicht mit Bezug auf die Teilhabe an existenznotwendigen Gütern und eine verfahrensrechtliche Pflicht, geeignete Untersuchungen in Diskriminierungsfällen durchzuführen. Während die Schutzpflicht das bekannte Problem aufwirft, dass drittgerichtete Diskriminierungsverbote immer mit Freiheitseinbußen verbunden sind, stellt sich mit Blick auf die Gewährleistungspflicht wiederum ein Einordnungsproblem: Die diskriminierungsfreie Teilhabe an Leistungen lässt sich wohl ebenso plausibel als Element traditionellen gleichheitsrechtlichen Abwehrschutzes darstellen, weil es den Gleichheitsrechten zunächst ganz egal ist, ob sich ihre modale Schutzwirkung gegen Ungleichbehandlungen im Eingriffs- oder im Leistungsbereich richtet. Hier hat sich eine konventionsrechtliche Sonderdogmatik herausgebildet, die vermutlich durch die Akzessorietät des Art. 14 EMRK und das heikle Verhältnis von EMRK und Sozialcharta bedingt ist.

4. Altwicker will abschließend die rechtsethischen Prinzipien untersuchen, die Recht und Praxis - so die nicht ganz glücklichen Überschriften des 2. und 3. Teils - des menschenrechtlichen Gleichheitsschutzes zugrunde liegen. Dieser Teil hat den Rezensenten etwas ratlos zurückgelassen. Zum einen wird hier mit recht viel Aufwand eine „rechtsethische Rekonstruktionsmethode“ vorgestellt, deren zusätzlicher Ertrag gegenüber den bereits üblichen Ansätzen, aus einem unübersichtlichen Rechtsmaterial leitende Prinzipien zu destillieren, sich nicht recht erschließt. Zum anderen wer- 
den dann in der Sache verschiedene Prinzipienangebote - Menschenwürde, Anerkennung, Inklusion, Effizienz, Gerechtigkeit - geprüft, ob und inwieweit sie zu der analysierten Rechtsprechung „passen“. Dabei gewinnt - wenig überraschend - die „Gerechtigkeit“, allerdings in einer speziellen, von Altwicker selbst entwickelten Variante: Es gehe um eine diskriminierungsfreie Gewährleistung der „Mittel des SoSein-Könnens von Personen“. Damit ist nun entweder etwas ziemlich Triviales gemeint, das kaum jemand bestreiten würde - dass nämlich die Diskriminierungsverbote die Funktion haben, die Teilhabe an Grundgütern zu gewährleisten -, oder etwas sehr Spezielles, das dann aber einer viel grundsätzlicheren Erläuterung und Begründung bedürfte. Und dass sich die einzelnen Gewährleistungsinhalte der Diskriminierungsverbote den Grundsätzen der korrektiven und distributiven Gerechtigkeit zuordnen lassen, wie Altwicker meint, wird auch nicht plausibel. Der Autor wollte hier einfach zu viel auf einmal.

\section{Fazit}

Insgesamt hinterlässt das Werk damit einen etwas ambivalenten Eindruck. Zwei grundsätzliche Einwände drängen sich auf. Zum einen ist die Zuordnung von dogmatischer Systematisierung (3. Teil) und rechtsethischer Rekonstruktion (4. Teil) wohl nicht ganz gelungen. Kann man das auf diese Weise - erst die Dogmatik, dann die Ethik - auseinanderreißen? Altwicker selbst kommt ja in seiner Systematisierung um Verweise nach unten nicht herum. Und so sollte es doch eigentlich auch sein: Die grundlegenden normativen Vorentscheidungen und Brüche müssen bei der Analyse der Dogmatik transparent gemacht und aus ihr begründet werden.

Zum anderen fällt es auf, dass Altwicker einen etwas hermetischen Zugang zur EGMR-Rechtsprechung hat. Schwärzte man den Namen des Gerichts, hätte der Leser kaum erkannt, dass es hier um die Rechtsprechung eines Menschengerichtshofs geht. Eine der Art nach identische Arbeit hätte sich auch über ein nationales Verfassungsgericht schreiben lassen. Dass vom EGMR Mitgliedstaaten und nicht gleichgeordnete Verfassungsorgane überprüft werden, spielt bei Altwicker für die Dogmatik praktisch keine Rolle. Anders gesagt: Die Gleichheitsfrage wird nicht als spezifisches - Ebenen- und Kompetenzproblem wahrgenommen. Altwicker schafft eine bewundernswerte Ordnung in der Gleichheitsschublade, aber er stellt nicht die Frage, ob alle Inhalte in diese Schublade wirklich hineingehören.

Und doch ist nochmals zu betonen, dass dieses Buch weit über das übliche Dissertationsniveau hinausgeht und dass man sich freuen würde, von diesem Autor alsbald wieder etwas zu lesen - allein schon deshalb, weil er hervorragend und sehr klar schreiben kann. Wer sich mit der Dogmatik der EMRK-Gleichheitsrechte intensiver beschäftigt, wird an dem zentralen 3. Teil dieses Buches nicht vorbeikommen. Beim Rest ist sich der Rezensent nicht so sicher. 